\title{
The Role of Tractography in Ischemic Stroke: A Review of the Literature
}

\author{
Alejandro Gonzalez-Aquines ${ }^{1}$, Talía Moreno-Andrade ${ }^{1 *}$, Fernando Gongora-Rivera ${ }^{1,2}$, \\ Adolfo C. Cordero-Perez ${ }^{1}$, Xóchitl Ortiz-Jiménez ${ }^{2,3}$, Oscar Cavazos-Luna ${ }^{1}$, Eduardo Garza-Villareal², \\ Mario Campos-Coy ${ }^{4}$ and Guillermo Elizondo-Riojas ${ }^{4}$
}

${ }^{1}$ Department of Neurology, School of Medicine, "Dr. José Eleuterio González" University Hospital, Autonomous University of Nuevo Leon, NL, Mexico; ${ }^{2}$ Neuroplasticity Unit, Centro de Investigacion y Desarrollo en Ciencias de la Salud, Autonomous University of Nuevo Leon, NL, Mexico; ${ }^{3}$ School of Psychology, Autonomous University of Nuevo Leon, NL, Mexico; ${ }^{4}$ Department of Radiology and Medical Imaging, School of Medicine, "Dr. José Eleuterio González" University Hospital, Autonomous University of Nuevo Leon, NL, Mexico

\begin{abstract}
Neuroimaging in acute stroke is needed to define the diagnosis, treatment, and establish the prognosis of the patient. Magnetic resonance and its different techniques have revolutionized neurology practice due to its greater definition of structures. Tractography is obtained by diffusion tensor imaging, which evaluates the diffusion of water molecules and creates an indirect representation of the integrity of white matter tracts. The use of tractography has focused on the prediction of motor outcome; however, its results are controversial. In this review, we explain how tractography is obtained and how the information is interpreted and translated into clinical practice. We discuss the role of tractography of the corticospinal and supplementary motor tracts in predicting the motor and functional outcome after acute stroke.
\end{abstract}

Key words: Diffusion tensor imaging. Tractography. Stroke. Motor recovery.

\section{Introduction}

Neuroimaging plays an important role in stroke diagnosis, treatment, and prognosis ${ }^{1}$. Since its beginning, magnetic resonance imaging (MRI) has contributed to a better understanding of the anatomy and function of the brain ${ }^{2,3}$. In a stroke, its role has progressed considerably and helps predict not only the functional outcome but also motor recovery ${ }^{4}$. MRI techniques provide useful information about the damage and temporality of the ischemic lesion ${ }^{5}$, thus helping to determine which patients are candidates for thrombolytic therapy ${ }^{6}$.

Diffusion tensor imaging (DTI) is an MRI technique that analyzes information from a diffusion-weighted imaging sequence and generates a three-dimensional representation of tissue through the evaluation of the diffusion of water molecules, thus performing an indirect evaluation of the integrity of white matter tracts? DTI has been used in stroke to measure the damage of motor tracts, mainly, the corticospinal tract (CST) ${ }^{8}$. Tractography of motor tracts provides valuable information of the white matter and helps assess the recovery of motor functions ${ }^{9}$, providing supplementary information for the management of poststroke patients.

In this narrative review, we discuss the basics of DTI tractography, the information gathered in recent years about the CST in stroke and its applications in predicting motor outcome as well as the different motor tracts that could influence poststroke recovery.

\section{Correspondence:}

Talia Moreno-Andrade

E-mail: taliamyr@hotmail.com
Available online: 18-01-2019

Date of reception: 07-08-2018

Date of acceptance: 08-11-2018

DOI: 10.24875/RMU.18000021
Medicina Universitaria. 2018;20(4):161-165 www.medicinauniversitaria.org CC BY-NC-ND license (http://creativecommons.org/licenses/by-nc-nd/4.0/). 


\section{How is a tractography obtained and what does it represent?}

Tractography was one of the first non-invasive methods to show white matter tract representations (Fig. 1). Initially, it was used to show white matter nerve tracts anatomically, and later, its applications were translated to the clinical setting, first in brain tumor evaluation and then in stroke ${ }^{10}$. The information needed to make a representation of white matter tracts is obtained from the DTI technique, which is an MRI technique that evaluates water molecules diffusion during a limited time frame. From this information, and using appropriate software, it is possible to create three-dimensional representations of white matter tracts ${ }^{11}$. A tensor is a mathematical entity with specific properties that allow complex physical phenomena to be quantified. The tensor is a matrix of numbers derived from diffusion measurements in various directions used to estimate the diffusibility in an arbitrary direction or to determine the direction of maximum diffusibility ${ }^{12}$.

DTI images measure the eigenvalues and eigenvectors of water molecules, which indicate the diffusion and direction of water molecules, respectively (Fig. 2). Diffusion is anisotropic (direction dependent) in the white matter tracts as the membranes, and the myelin sheaths represent a barrier for water molecules and limit their movement. The maximum diffusivity direction indicates the orientation of the water molecules, therefore, establishing the path of the white matter tract ${ }^{13}$.

Quantitative measures are obtained from the DTI, such as fractional anisotropy (FA), axial diffusivity (AD), and radial diffusivity (RD), providing objective measures to evaluate changes after brain damage. FA is obtained from the eigenvalues and represents the diffusivity of water molecules in a specific region ${ }^{14}$. FA ranges from 0 to 1 , in which sphere-shaped molecules have a value of 0 , and ellipsoid-shaped molecules have a value closer to 1 . In the context of the integrity of white matter tracts, an FA closer to 1 represents better integrity, whereas when its value is closer to 0 , it represents damage or disruption of the evaluated tract. $A D$ is obtained from the main eigenvector, and the two left eigenvectors are used to calculate RD.

A good spatial resolution is necessary for the evaluation of white matter tracts; hence, cubic voxels of $2.5 \mathrm{~mm}$ or less are recommended to obtain the eigenvalues and eigenvectors from the area of interest ${ }^{8}$. Brain white matter is characterized by its high connectivity between tracts, for example, in associative areas; therefore, anisotropy and the orientation of fibers are used

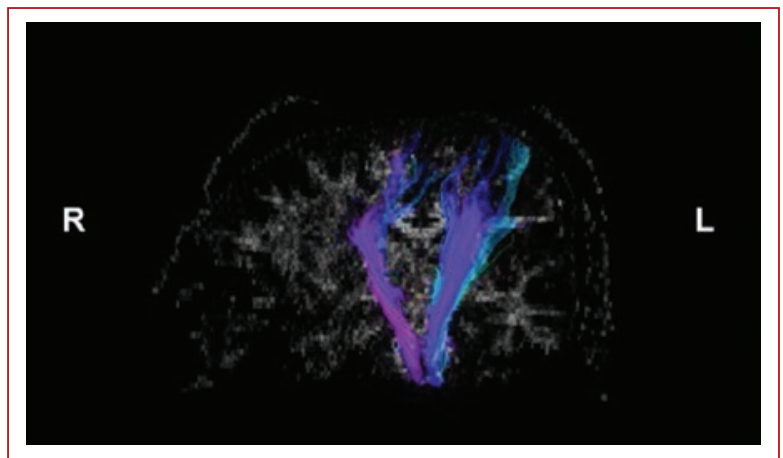

Figure 1. Corticospinal tract of an acute ischemic stroke of the right middle cerebral artery.

R: right; L: left

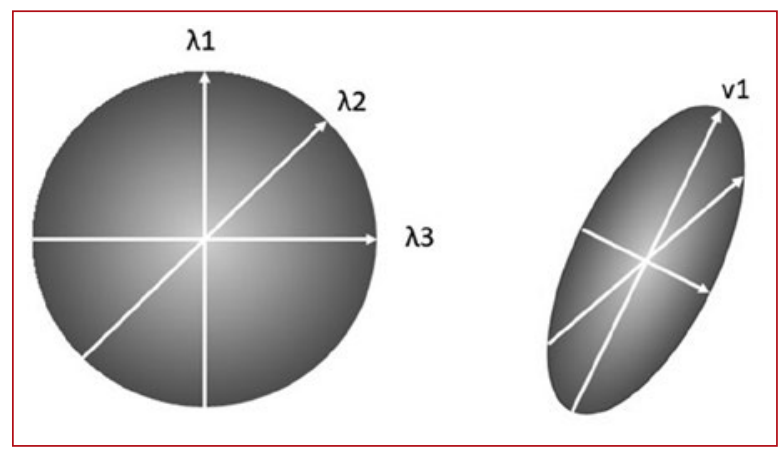

Figure 2. Left. The eigenvalues $(\lambda 1, \lambda 2$, and $\lambda 3)$ indicate the diffusion of water molecules. When there is no diffusion, the fractional anisotropy is zero and has a spherical shape. Right. The eigenvector (v1) determines the direction in which the water molecules travel. Together, eigenvectors and eigenvalues establish the shape and direction of water molecules.

as measures to differentiate between the different tracts. Furthermore, using this information, tractography can analyze white matter tracts through different methods such as the region of interest (ROI), histogram, or voxel-based analysis. We will discuss ROI analysis, as it is the one most used in the clinical setting.

$\mathrm{ROI}$ analysis needs previous anatomical knowledge as the way to create a representation of the tracts by selecting seed points, specific regions where the tract of interest travels (Fig. 3). After placing seed points in $\mathrm{ROI}$, tractography could be generated by two algorithms: deterministic and probabilistic. In deterministic tractography, voxels are connected to neighboring voxels that meet specific criteria such as a concordant anisotropy or angulation, until the end of the tract. On the other hand, probabilistic tractography generates a 


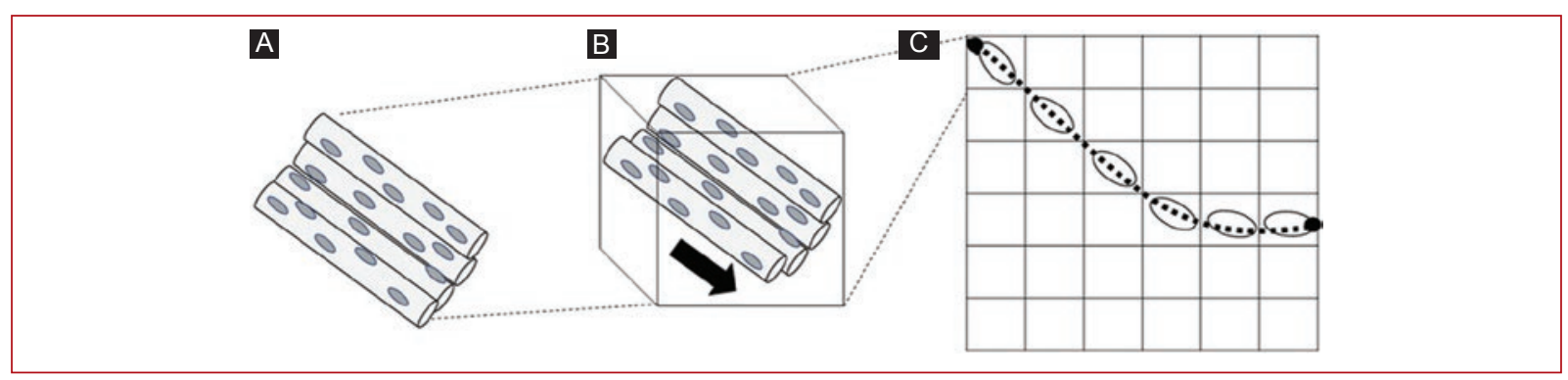

Figure 3. (A) Representation of axons. The water molecules (oval-gray shapes) have an oval structure and a limited space to move. (B) Representation of a voxel. Water molecules present in a defined space are measured, and diffusion is calculated based on fractional anisotropy (FA) values. (C) Analysis of the direction of the fibers. Once the regions of interest (black points) are established, FA values are measured, and the white matter tract is followed (dotted line).

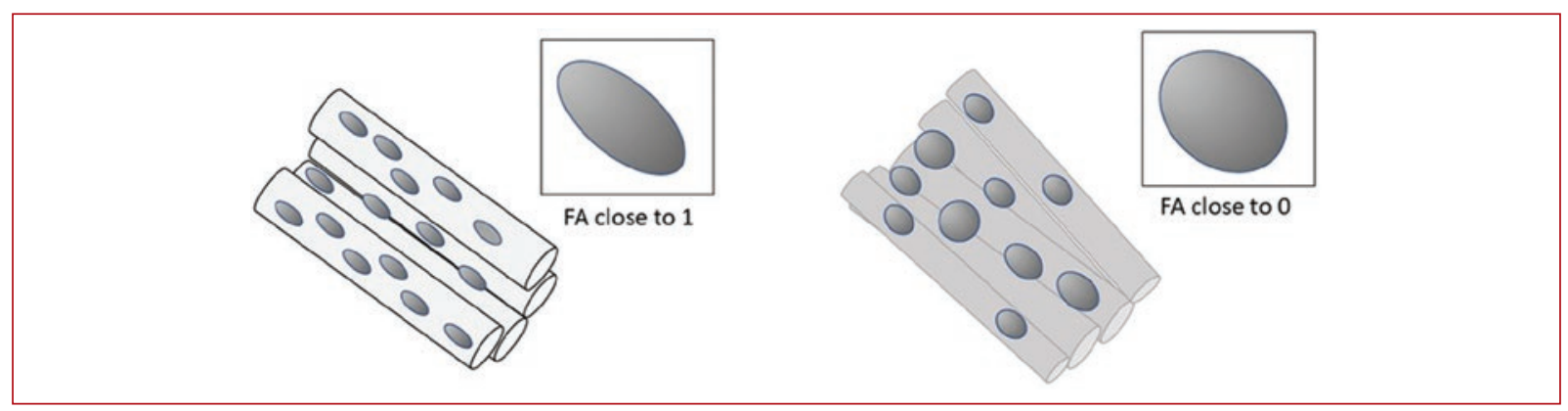

Figure 4. Left. Normal integrity of the axons. Due to the limited space to move freely, fractional anisotropy (FA) is closer to 1. Right. After a stroke, there is a deterioration of the axons. Water molecules take a different shape, decrease and their diffusivity changes, diminishing the FA value.

whole map between both seed points and calculates the probability of one voxel to follow an adjacent voxel15,16.

\section{Tractography in stroke: the CST}

Despite the developments in the medical management of stroke, poststroke recovery remains a challenge. Functional and motor evolution after stroke is characterized by spontaneous neurological recovery during the $1^{\text {st }}$ week, where clinical and neuroimaging information at baseline have been studied as predictive factors ${ }^{17}$. Tractography has been used to track the anatomical correlates of poststroke recovery and has shown a high reproducibility ${ }^{18}$. One of the most studied white matter tracts is CST, which is also the main motor tract and the most affected in stroke. To evaluate the integrity of CST, seed points could be placed in the posterior limb of the internal capsule and in the lower pons to generate a representation of CST.

In recent decades, FA and $A D$ values from the CST have been correlated with long-term motor and functional outcomes, providing information about the applications of DT tractography in clinical practice. After a stroke, a series of changes in the microarchitecture of the brain occur and produce a deterioration of axon integrity. The results of this altered integrity change the shape of water molecules and axons resulting in a decrease in FA (Fig. 4).

Despite the advances in DT tractography, different results have been obtained depending on the time of evaluation. Whereas some reports mention the lack of predictability of $F A$ in the acute period ${ }^{19}$, there are others that have found an association between FA and motor outcome ${ }^{20}$, especially after rehabilitation therapy ${ }^{21}$. On the other hand, the evaluation of FA in the subacute or chronic period of stroke correlated with motor function $^{22,23}$ and had a predictive value for motor recovery $^{19,22,24}$. A meta-analysis by Kumar et al. revealed a correlation between the FA values during the subacute period with upper limb motor recovery (Correlation Coefficient $=0.82 ; 95 \% \mathrm{Cl} 0.66-0.90)^{25}$. Furthermore, the integrity of CST, categorized as present or absent, is 
also able to predict the motor outcome ${ }^{26}$. Changes after stroke can also be evaluated by measuring FA ratio $(\mathrm{rFA})$, which is calculated by dividing FA of the ipsilesional hemisphere by FA from the contralesional hemisphere. A decrease in FA in the affected hemisphere and $\mathrm{rFA}$ of the ipsilesional side is associated with a poorer motor outcome $\mathrm{e}^{15,20}$. Interestingly, it has also been suggested that the contralesional side participates in motor outcome ${ }^{27}$.

The debate of the ability of FA in the acute period led to a search for earlier values obtained from DT tractography that could have a greater correlation with motor recovery. Therefore, AD, which is also affected after a stroke, has proven to be more sensitive in the acute period ${ }^{28}$. Although experience evaluating $A D$ is limited and controversy exists about the reliability of this value ${ }^{29}$, results from diverse studies have provided information about the correlation of decreased $A D$ values with a worse long-term motor function ${ }^{22,28}$.

After a stroke, most patients remain with a motor disability and rehabilitation should be initiated in hospital and continued after hospital discharge ${ }^{30}$. Nonetheless, it remains unclear which patients benefit the most and with what intensity of rehabilitation. Therefore, changes in FA values have also been evaluated after rehabilitation and it has been demonstrated that a positive change of the affected CST correlates with an improvement in motor outcome ${ }^{24,31}$. In one of these studies, CST and the transcallosal tract were evaluated with both showing a strong relationship between the increased FA and a better outcome, suggesting that other tracts besides the CST are involved in the recovery after stroke.

\section{Tractography in stroke: supplementary motor tracts}

Enough evidence exists about the role of CST in poststroke recovery; however, CST establishes connections with fibers originating from different regions within the brain; therefore, the tracts formed from these fibers could have an important role in motor outcome after stroke.

Secondary motor areas (SMAs) contribute to motor recovery, possibly due to compensatory functions and support to primary areas. ${ }^{32}$ Schulz et al. evaluated the cerebellar connections with the CST, showing that the integrity of the cortico-ponto-cerebellar tract and the dento-thalamo-cortical tract was associated with a better motor outcome and fine motor skills ${ }^{33}$. Another well-recognized tract is the rubrospinal tract, which originates in the red nucleus and has indirect connections with the main motor cortex. After evaluating efferent neurons from the red nucleus and realizing a voxel-wise analysis of FA values, motor function was compared with the contralesional side and healthy controls, finding a strong relation between the increasing FA in the vicinity of the red nucleus and motor function $^{34}$. Even in longitudinal observations, it has been evidenced that the rubrospinal tract influences motor recovery ${ }^{35}$.

Schulz et al. demonstrated that the function of alternate corticofugal motor fibers contributes independently to motor function ${ }^{23}$. Nevertheless, results from SMA have also been reported as inconclusive due to inconsistency in motor outcomes ${ }^{36}$. More information about different motor tracts other than CST is needed to perform a comprehensive evaluation of the motor prognosis after acute stroke.

\section{Conclusions}

Evidence of the role of tractography in stroke has increased in the past decades. Changes in the microstructure in the central nervous system after acute stroke translate into lower values of FA and continue to drop in the subacute phase of stroke, where its correlation with motor scales is higher. Rehabilitation fosters motor improvement and an increase in FA values, which suggest a remodeling of white matter motor tracts. Further, research of CST and supplementary motor areas by diffusion tensor tractography must be done with standardized motor scales to have a better evaluation and comprehension of poststroke evolution.

\section{Acknowledgments}

The authors acknowledge the support provided by the Department of Neurology and CIDICS, for the development of the study.

\section{Conflicts of interest}

The authors declare no financial support and no conflicts of interest.

\section{Funding}

No funding was received for the development of the study. 


\section{Ethical disclosures}

Protection of human and animal subjects. The authors declare that no experiments were performed on humans or animals for this study.

Confidentiality of data. The authors declare that no patient data appear in this article.

Right to privacy and informed consent. The authors declare that no patient data appear in this article.

\section{References}

1. Jauch EC, Saver JL, Adams HP Jr, et al. Guidelines for the early management of patients with acute ischemic stroke: a guideline for healthcare professionals from the american heart association/American stroke association. Stroke. 2013:44:870-947.

2. Pukenas B. Normal brain anatomy on magnetic resonance imaging. Magn Reson Imaging Clin N Am. 2011;19:429-37.

3. Ward NS, Swayne OB, Newton JM. Age-dependent changes in the neural correlates of force modulation: an fMRI study. Neurobiol Aging. 2008;29:1434-46.

4. Takahashi CD, Yeghiaian DL, Cramer SC. Stroke recovery and its imaging. Neuroimaging Clin N Am. 2005;15:681-95.

5. Lövblad KO, Altrichter S, Pereira MV, et al. Imaging of acute stroke: ct and/or MRI. J Neuroradiol. 2015;42:55-64.

6. Sanossian N, Fu KA, Liebeskind DS, et al. Utilization of emergent neuroimaging for thrombolysis-eligible stroke patients. J Neuroimaging. 2017;27:59-64

7. Lerner A, Mongansen MA, Kim PE, et al. Clinical applications of diffusion tensor imaging. World Neurosurg. 2014;82:96-109.

8. Mukherjee P. Diffusion tensor imaging and fiber tractography in acute stroke. Neuroimaging Clin N Am. 2005;15:655-5.

9. Puig J, Blasco G, Schlaug G, et al. Diffusion tensor imaging as a prognostic biomarker for motor recovery and rehabilitation after stroke. Neuroradiology. 2017;59:343-51

10. Mori S, Frederiksen K, Van Zijl PC, et al. Brain white matter anatomy of tumor patients evaluated with diffusion tensor imaging. Ann Neurol. 2002 51:377-80.

11. Kunimatsu A, Aoki S, Masutani $Y$, et al. Three-dimensional white matter tractography by diffusion tensor imaging in ischaemic stroke involving the corticospinal tract. Neuroradiology. 2003;45:532-5.

12. Jellison BJ, Field AS, Medow J, et al. Diffusion tensor imaging of cerebral white matter: a pictorial review of physics, fiber tract anatomy, and tumor imaging patterns. Am J Neuroradiol. 2004;25:356-69.

13. Moseley ME, Cohen Y, Kucharczyk J, et al. Diffusion-weighted MR imaging of anisotropic water diffusion in cat central nervous system. Radiology. 1990;176:439-45.

14. O'Donnell LJ, Westin CF. An introduction to diffusion tensor image analysis. Neurosurg Clin N Am. 2011;22,185-96.

15. Rong $\mathrm{D}$, Zhang M, Ma Q, Lu J, Li K. Corticospinal tract change during motor recovery in patients with medulla infarct: a diffusion tensor imaging study. Biomed Res Int. 2014;2014:524096.
16. Nucifora PG, Verma R, Lee SK, Melhem ER. Diffusion-tensor MR imaging and tractography: exploring brain microstructure and connectivity. Radiology. 2007;245:367-84.

17. Stinear CM. Prediction of motor recovery after stroke: advances in biomarkers. Lancet Neurol. 2017;16:826-36.

18. Lin CC, Tsai MY, Lo YC, et al. Reproducibility of corticospinal diffusion tensor tractography in normal subjects and hemiparetic stroke patients. Eur J Radiol. 2013;82:e610-6.

19. Kwon YH, Jeoung YJ, Lee J, et al. Predictability of motor outcome according to the time of diffusion tensor imaging in patients with cerebral infarct. Neuroradiology. 2012:54:691-7.

20. Møller M, Frandsen J, Andersen G, et al. Dynamic changes in corticospinal tracts after stroke detected by fibretracking. J Neurol Neurosurg Psychiatry. 2007;78,587-92.

21. Radlinska B, Ghinani S, Leppert IR, et al. Diffusion tensor imaging, permanent pyramidal tract damage, and outcome in subcortical stroke. Neurology. 2010;75:1048-54.

22. Groisser BN, Copen WA, Singhal AB, Hirai KK, Schaechter JD. Corticospinal tract diffusion abnormalities early after stroke predict motor outcome. Neurorehabil Neural Repair. 2014;28:751-60.

23. Schulz R, Park E, Lee J, et al. Synergistic but independent: the role of corticospinal and alternate motor fibers for residual motor output after stroke. Neuroimage Clin. 2017;15:118-24.

24. Fan YT, Lin KC, Liu HL, Chen YL, Wu CY. Changes in structural integrity are correlated with motor and functional recovery after post-stroke rehabilitation. Restor Neurol Neurosci. 2015;33:835-44.

25. Kumar $\mathrm{P}$, Kathuria $\mathrm{P}$, Nair $\mathrm{P}$, Prasad K. Prediction of upper limb motor recovery after subacute ischemic stroke using diffusion tensor imaging: a systematic review and meta-analysis. J Stroke. 2016;18:50-9.

26. Cho SH, Kim DG, Kim DS, et al. Motor outcome according to the integrity of the corticospinal tract determined by diffusion tensor tractography in the early stage of corona radiata infarct. Neurosci Lett. 2007:426:123-7.

27. Seitz RJ, Donnan GA. Role of neuroimaging in promoting long-term recovery from ischemic stroke. J Magn Reson Imaging. 2010;32:756-72.

28. Moulton E, Amor-Sahli M, Perlbarg V, et al. Axial diffusivity of the corona radiata at 24 hours post-stroke: a new biomarker for motor and global outcome. PLoS One. 2015;10:e0142910.

29. Wheeler-Kingshott AM, Cercignani M. About 'axial' and 'radial' diffusivities. Magn Reson Med. 2009:61:1255-60.

30. Winstein CJ, Stein J, Arena R, et al. Guidelines for adult stroke rehabilitation and recovery: a guideline for healthcare professionals from the American heart association/American stroke association. Stroke. 2016; 47:e98-169.

31. Wen $\mathrm{H}$, Alshikho MJ, Wang $\mathrm{Y}$, et al. Correlation of fractional anisotropy with motor recovery in patients with stroke after postacute rehabilitation. Arch Phys Med Rehabil. 2016;97:1487-95.

32. Schulz R, Park $\mathrm{CH}$, Boudrias $\mathrm{MH}$, et al. Assessing the integrity of corticospinal pathways from primary and secondary cortical motor areas after stroke. Stroke. 2012:43:2248-51.

33. Schulz R, Frey BM, Koch P, et al. Cortico-cerebellar structural connectivity is related to residual motor output in chronic stroke. Cereb Cortex. 2017; $27: 635-45$

34. Rüber T, Schlaug $\mathrm{G}$, Lindenberg $\mathrm{R}$. Compensatory role of the cortico-rubro-spinal tract in motor recovery after stroke. Neurology. 2012;79:515-22.

35. Takenobu $\mathrm{Y}$, Hayashi $\mathrm{T}$, Moriwaki $\mathrm{H}$, et al. Motor recovery and microstructural change in rubro-spinal tract in subcortical stroke. Neuroimage Clin. 2014;4:201-8.

36. Phan TG, Van Voort S, Chen J, et al. Impact of corticofugal fibre involvement in subcortical stroke. British Med J Open. 2013;3:e003318. 